\title{
A study of L-dependent Pc3 pulsations observed by low Earth orbiting CHAMP satellite
}

\author{
D. C. Ndiitwani and P. R. Sutcliffe \\ Hermanus Magnetic Observatory, P.O. Box 32, Hermanus, 7200, South Africa \\ University of Kwazulu-Natal, Private Bag X54001, Durban, 4000, South Africa
}

Received: 2 November 2009 - Revised: 24 December 2009 - Accepted: 22 January 2010 - Published: 3 February 2010

\begin{abstract}
Field line resonances (FLR) driven by compressional waves are an important mechanism for the generation of ULF geomagnetic pulsations observed at all latitudes during local daytime. References to observations of toroidal standing Alfvén mode oscillations with clearly L-dependent frequencies from spacecraft in the outer magnetosphere for $L>3$ are limited in the literature. Such observations in the inner magnetosphere for $L<3$ have not yet been reported in the literature. This study offers two interesting case studies of observations of ULF waves by the low Earth orbiting CHAMP satellite. The magnetic field measurements from CHAMP, which are of unprecedented accuracy and resolution, are compared to Hermanus magnetometer data for times when CHAMP crosses the ground station L-shell, namely for 13 February 2002 and 18 February 2003. The data were analysed for Pc3 pulsation activity using the Maximum Entropy Spectral Analysis (MESA) method to visualise FLRs in the vector magnetometer data. For the first time observations of Pc3 toroidal oscillations with clearly L-dependent frequencies for lower $L$-shell values $(L<3)$ observed by an LEO satellite are reported. These observations show FLR frequencies increasing as a function of decreasing latitude down to $L=1.6$ and then decreasing as a result of the larger plasma density of the upper ionosphere. The L-dependent frequency oscillations were observed in the presence of a broadband compressional wave spectrum. Our observations thus confirm the well-known magnetohydrodynamic (MHD) wave theoretical prediction of a compressional wave being the driver of the field line resonance.
\end{abstract}

Keywords. Magnetospheric physics (Magnetosphereionosphere interactions; MHD waves and instabilities; Solar wind-magnetosphere interactions)

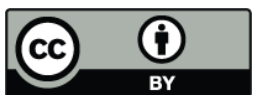

Correspondence to: D. C. Ndiitwani (cndiitwani@hmo.ac.za)

\section{Introduction}

Magnetometers on board spacecraft and on the Earth's surface detect the magnetic signature of ultra-low frequency (ULF) geomagnetic field line resonances known as geomagnetic pulsations. Geomagnetic pulsations are characterized by their period and structure as continuous Pc or irregular Pi pulsations (Jacobs et al., 1964). The continuous pulsations such as Pc3 are mainly thought to be the resonances of either geomagnetic field lines with ends fixed in conjugate ionospheres or of cavities in the magnetosphere (Dungey, 1954, 1964). The resonance mechanism follows three steps: the source wave is excited at or near the dayside magnetopause/bow shock, it propagates into the magnetosphere as a fast-mode magnetosonic wave, and the latter couples into the local standing Alfvén wave (Southwood, 1974; Chen and Hasegawa, 1974). The treatment of pulsations as harmonics of Alfvén waves follows suggestions (Sugiura and Wilson, 1964) that field lines or shells oscillate independently from each other in a manner similar to that of resonant waves on a vibrating string.

The Earth's foreshock, the region between the tangential interplanetary magnetic field (IMF) line and the bow shock, is filled with backstreaming electrons and ions (Le and Russel, 1996; Shin et al., 2008). Observations suggest that ULF waves generated in the region upstream of the bow shock are moved downstream by the solar wind into the magnetosheath by convection. If the IMF cone angle is small, waves in the subsolar upstream region are carried to the magnetopause boundary. The magnetopause responds to these pressure fluctuations and transfers wave energy into the dayside magnetosphere where they can excite field line resonances (Le and Russell, 1994). It is generally agreed that the ultimate source of dayside Pc3 and Pc4 geomagnetic pulsations are upstream waves.

The characteristics of field line resonant oscillations excited by fast mode waves may be expected to depend on

Published by Copernicus Publications on behalf of the European Geosciences Union. 
the source wave frequency content, that is, whether it is monochromatic or broad-band. Magnetic pulsations can be excited when a monochromatic source is present in the magnetosphere and the field line with eigenperiod similar to the monochromatic source resonates with maximum intensity at the L-shell value of the resonant field line (Chen and Hasegawa, 1974; Southwood, 1974). Hasegawa et al. (1983) present a theoretical discussion that supports the conjecture of a wideband source able to excite harmonics for a given field line. The Hasegawa mathematical model explains how the local field line can oscillate at its resonant frequency in response to a wideband source, the frequency spectrum of which covers the resonant frequency. This explains certain observations of magnetic pulsations where the frequency varies continuously as a function of latitude for a given event. In this study this model is employed to interpret the Ldependence of FLR frequencies for Pc3 pulsations observed on L-shells with $L<3$. This kind of FLR structure has previously been observed in the outer magnetosphere between $L=3$ and 6 by Takahashi et al. (1990) and between $L=4.5$ and 9.1 by Engebretson et al. (1987).

In order to remedy the lack of observations of Pc3 magnetic pulsations in the outer magnetosphere, Takahashi et al. (1990) initiated an analysis of magnetic field data between $L=2$ and 6 acquired by the AMPTE CCE spacecraft. They presented dayside pulsation activity recorded by CCE during inbound passes. The noise level of AMPTE CCE data was at least 4 times stronger for $L<2.3$ than for $L>2.3$, while the amplitude of pulsations is expected to decrease at smaller L-shells, thus making it relatively difficult to detect pulsations at $L<2.3$. The ability to detect Pc3-4 pulsations was made possible by averaging the highest time resolution $(0.124 \mathrm{~s})$ data down to nearly exactly the spacecraft spin period $(5.9 \mathrm{~s})$. They identified toroidal standing Alfvén wave oscillations with clearly L-dependent frequencies between $L=3$ and 6 . Their ability to detect small amplitudes in CCE magnetometer data lead to the possibility of identifying the origin of various waves types of magnetic pulsation observed on the ground at low and middle latitudes using in situ measurements by satellites. They generated dynamic spectra using the FFT method computed for a 6-min window progressively shifted by $1.5 \mathrm{~min}$ at each step. The dynamic spectra were characterized by traces of pulsation activity with frequency that falls with $L$ between $L=3$ and 6 . Engebretson et al. (1987) observed toroidal oscillations with frequencies that decreased as a function of increasing L-shell from $L=4.5$ up to 9.1 and that increased again as the satellite moved to lower L-shells for a single full orbit.

Menk et al. (2000) utilized a temporary 12 magnetometer ground array in Eastern Australia spanning $L=1.3$ to 2.0 to investigate the variation in $\mathrm{Pc} 3-4$ power with latitude, the nature and low-latitude limit of FLRs, and properties of spectral components below the local resonant frequency. They employed a number of effective methods for detecting FLRs such as those described by Baransky et al. (1985, 1989) and Waters et al. (1991, 1994, 1995). They found that the resonant frequency increases with decreasing latitude up to $L \sim 1.6$ and then decreases at lower latitudes as a result of mass loading on the field line eigenfrequency due to the effect of ionospheric heavy ions at low altitudes (Hattingh and Sutcliffe, 1987).

Vellante et al. (2004) used SEGMA and CHAMP data to show that discrete Pc3 FLR oscillations are excited at low latitudes where the local resonant frequency matched that of the fast mode driver. Ndiitwani and Sutcliffe (2009) confirmed this observation using an independent data set to study the structure of low-latitude Pc3 pulsations observed by CHAMP and on the ground. In the current study, we continue the investigation of toroidal oscillations at $L<3$ observed by CHAMP. However, we find that the spectral structure of the FLR oscillations differs from that reported in the previous two papers. The current results are consistent with Hasegawa's mathematical model (Hasegawa et al., 1983). They are also consistent with previous satellite observations in the outer magnetosphere from $L=3$ up to 6 by Takahashi et al. (1990) and between $L=4.5$ and 9.1 by Engebretson et al. (1987). Some characteristics of the spectral structure also agree with the statistical results determined from ground observations by Menk et al. (2000). To the best of our knowledge, this study is the first to report on toroidal resonant oscillations observed at $L<3$ with clearly L-dependent frequencies, which vary continuously with latitude and are resolved from LEO satellite vector magnetometer data. Takahashi et al. (1990) had to adopt special techniques to detect Pc3-4 pulsations with amplitudes of only $1 \mathrm{nT}$ or less. In this study, CHAMP magnetic field data of unprecedented resolution are used and make it possible to extract and resolve clear Pc3 oscillations from the data.

The magnetometer data are analysed primarily using the Maximum Entropy Spectral Analysis (MESA) technique first proposed by Burg $(1967,1968)$. MESA sometimes suffers anomalous behaviour, such as frequency shifts, when the Burg (1968) algorithm is used to compute the prediction error filter (PEF) coefficient (Chen and Stegn, 1974). Consequently, we used Ulrych and Clayton's (1976) least squares estimator algorithm to compute the (PEF) coefficients, which obviates the shortcomings of the Burg (1968) algorithm. The PEF orders established by Ndiitwani and Sutcliffe (2009) experimenting with artificially generated signals, were employed. In order to identify FLRs in ground-based and satellite data PEF orders of 15 and 20, respectively, are regarded as adequate.

\section{Observations}

In this study we present an analysis of the $\mathrm{Pc} 3$ pulsation events of 13 February 2002 and 18 February 2003 for times when the CHAMP satellite crossed the Hermanus (HER) ground station L-shell. Figure 1 shows a map of the southern 


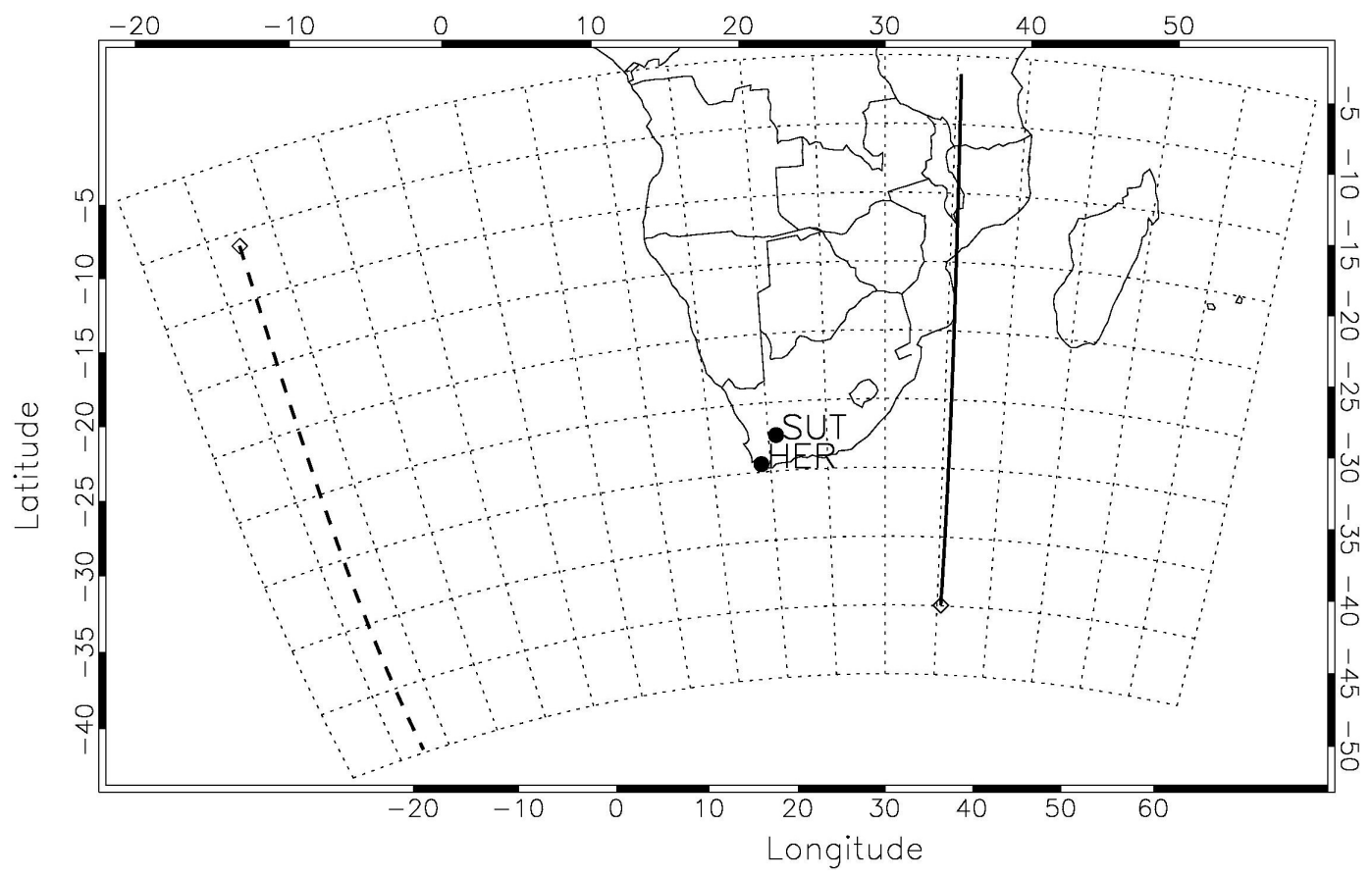

Fig. 1. Map of southern African region that also extends to central Africa showing the satellite ground tracks as it traversed the region during Pc3 pulsation activities of 13 February 2002 (solid line) and 18 February 2003 (dashed line). The locations of Hermanus and Sutherland ground stations are also shown.

African and adjacent ocean regions indicating the locations of two induction magnetometers for recording geomagnetic pulsations in South Africa, namely at Hermanus (HER) $\left(34^{\circ} 25^{\prime} \mathrm{S}, 19^{\circ} 13^{\prime} \mathrm{E}\right)$ and Sutherland (SUT) $\left(32^{\circ} 24^{\prime} \mathrm{S}\right.$, $20^{\circ} 40^{\prime} \mathrm{E}$ ). This station pair can be used to facilitate field line resonance detection using the amplitude difference method of Baransky et al. (1985) and cross phase technique of Waters et al. (1991, 1994). The two lines across the map show the CHAMP ground tracks during the two traverses, the respective paths on 13 February 2002 and 18 February 2003 indicated by solid and dashed lines on the map. The first points during the considered times are indicated by diamond symbols. The solid line shows CHAMP moving in the direction of the equator, whereas the dashed line shows the satellite moving poleward. In both cases the satellite covered almost 40 degrees of latitude in $10 \mathrm{~min}$ due to the low Earth orbit, thus a rapid movement of the satellite across different L-shell regions. The continuous movement of CHAMP across these L-shells provides an advantage to study the latitude dependence of field line resonance frequency.

Since the prime objective of this paper is to study FLRs, we use well established techniques to demonstrate the presence of FLRs in the ground-based data prior to considering them in combination with the satellite data. Figure 2 shows the amplitude difference and cross phase plots for the event on 13 February 2002 (discussed in detail below) that occurred between 07:25 and 07:35 UTC. The top panel

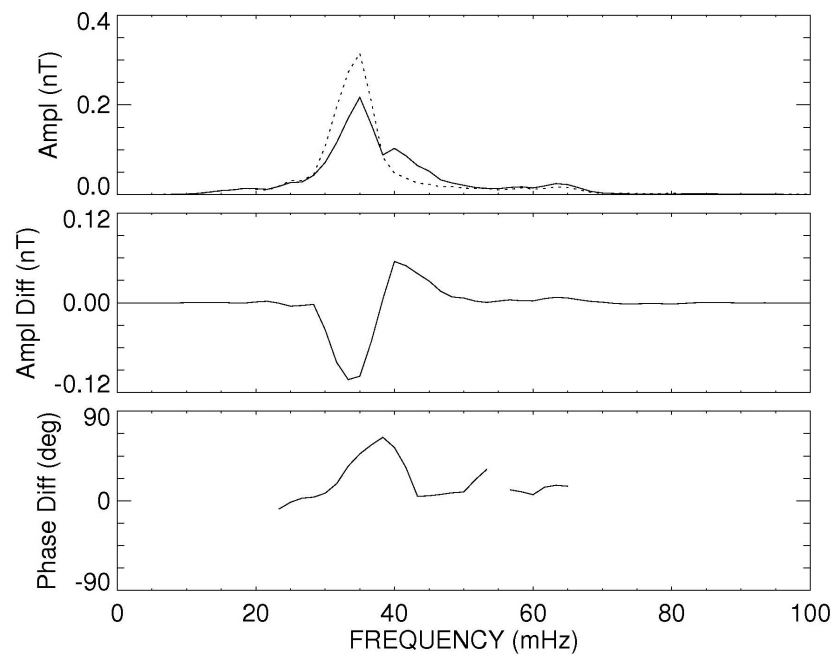

Fig. 2. The spectrum of the Pc3 pulsation H-component observed on the ground for the 12 February 2002 event that occurred between 07:25 and 07:35 UTC. Top panel: Amplitude spectra at HER and SUT, Middle panel: SUT-HER amplitude difference and bottom panel: SUT-HER phase difference.

shows the amplitude spectra at HER (dotted line) and SUT (solid line). The HER spectrum exhibits a peak centered at $\sim 35 \mathrm{mHz}$. The SUT spectrum exhibits two peaks at $\sim 35$ and 
(a)

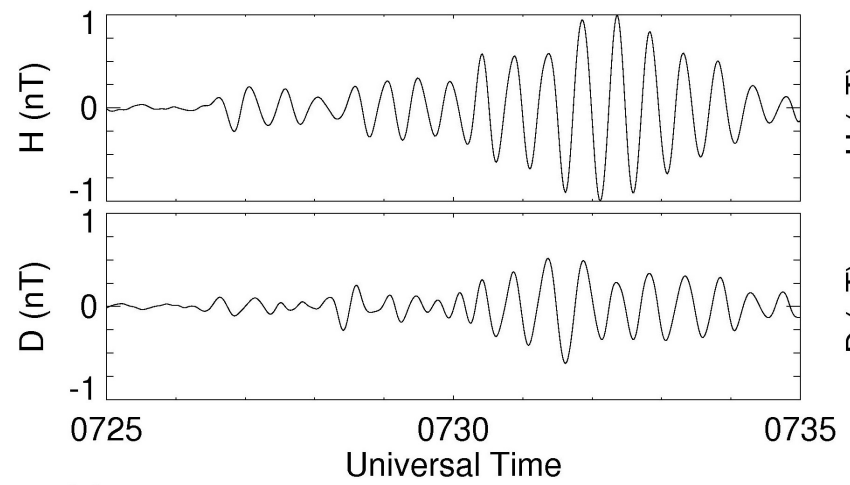

(c)

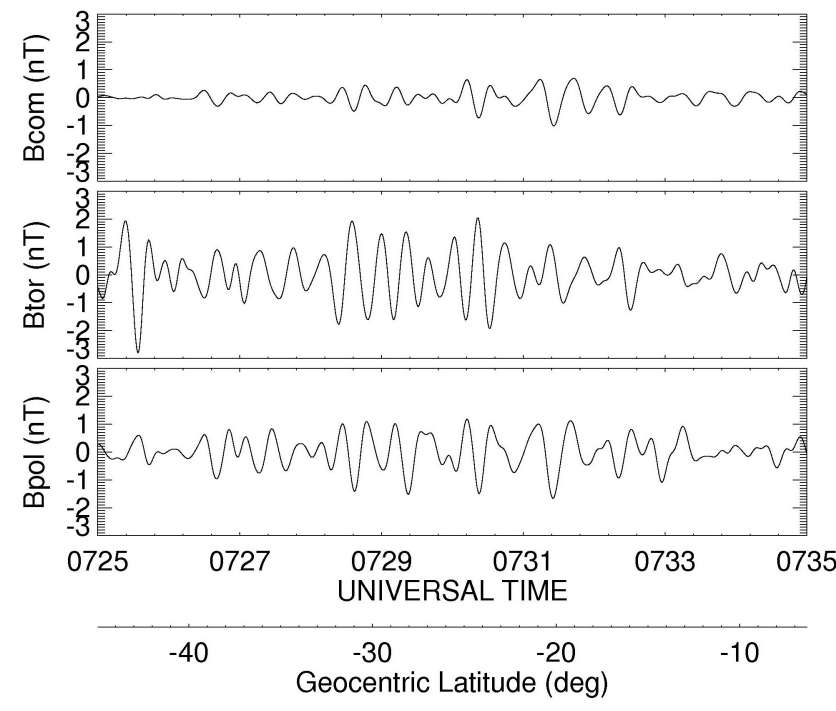

(b)

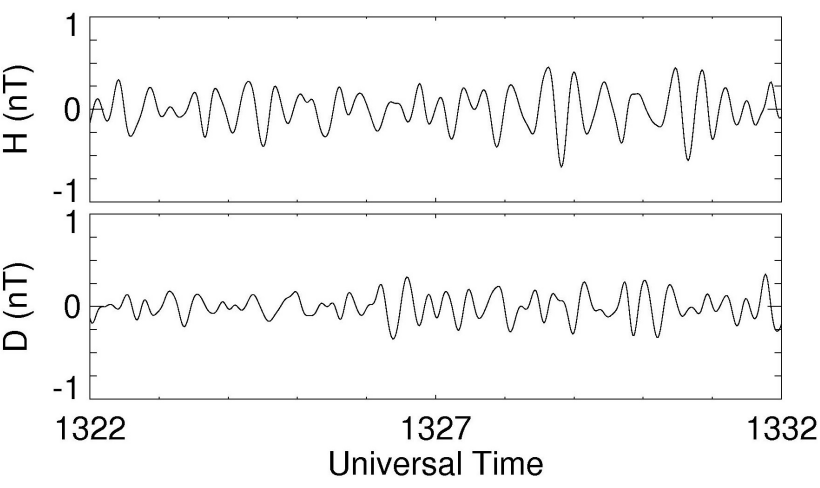

(d)

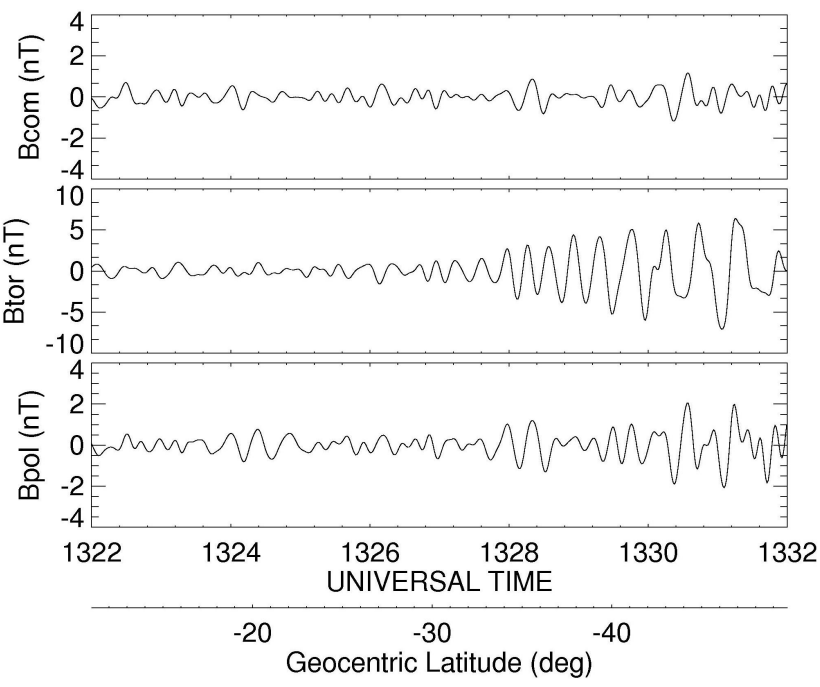

Fig. 3. The pulsation signals observed simultaneously on ground and along CHAMP trajectory. H- and D-components of the Pc3 pulsation activities observed on the ground on (a) 13 February 2002 and (b) 18 February 2003. Two Pc3 signals observed along the CHAMP satellite trajectories on (c) 13 February 2002 and (d) 18 February 2003. The CHAMP vector magnetic components shown are in a field-aligned coordinate system i.e. compressional $\left(B_{\mathrm{com}}\right)$, toroidal $\left(B_{\mathrm{tor}}\right)$ and poloidal $\left(B_{\text {pol }}\right)$, from top to bottom.

$\sim 42 \mathrm{mHz}$. The bottom two plots are the SUT-HER amplitude and phase difference, respectively. Both amplitude and phase difference techniques identify FLR occurring close to $37 \mathrm{mHz}$. This is in agreement with the dynamic cross-phase spectrum (not shown) that indicated FLR frequency of 40$35 \mathrm{mHz}$ between 06:00 and 18:00 UTC.

\subsection{Event 1}

The first event considered occurred on 13 February 2002 between 07:25-07:35 UTC. During this time a Pc3 was simultaneously observed on the ground at HER and in the upper ionosphere along the CHAMP trajectory as shown in Fig. 3a and c, respectively. Figure 3a shows $\mathrm{H}$ - and D-components as measured by the Hermanus ground station. The com- ponents in a field-aligned coordinate system observed by CHAMP are shown in Fig. 3c. In this coordinate system the compressional $\left(B_{\text {com }}\right)$ component is aligned with the ambient magnetic field direction (positive North), the toroidal ( $\left.B_{\text {tor }}\right)$ component represents the azimuthal perturbation (positive East), and the poloidal ( $\left.B_{\mathrm{pol}}\right)$ component lies in the magnetic meridian plane (positive inward). Also shown is the geocentric latitude axis which illustrates the change of latitude over a given time. These time series signals were filtered in the Pc3 frequency band, $20-100 \mathrm{mHz}$. The ground signal appears monochromatic and the H-component has a larger amplitude than the D-component. The satellite data appears to be more broadband. The $B_{\text {tor }}$ exhibits larger amplitude than both the $B_{\text {com }}$ and $B_{\text {pol }}$ components. 
(a)

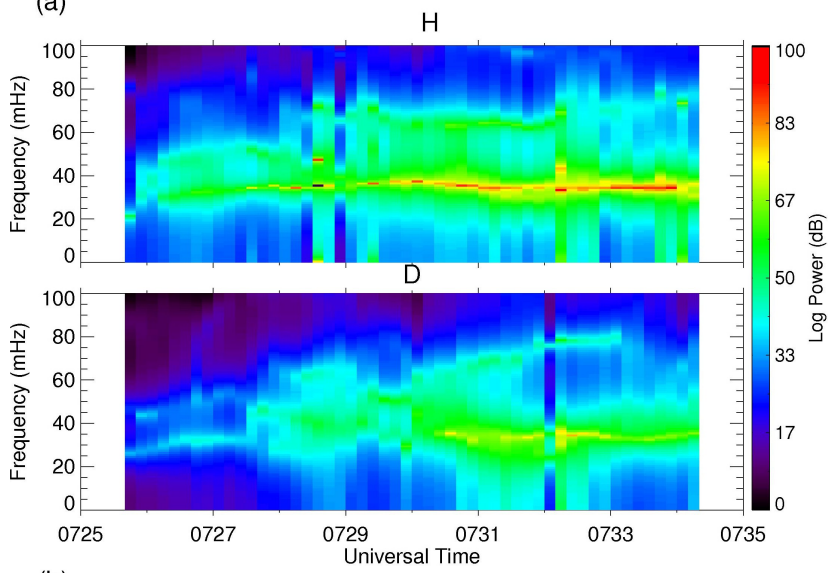

(b)

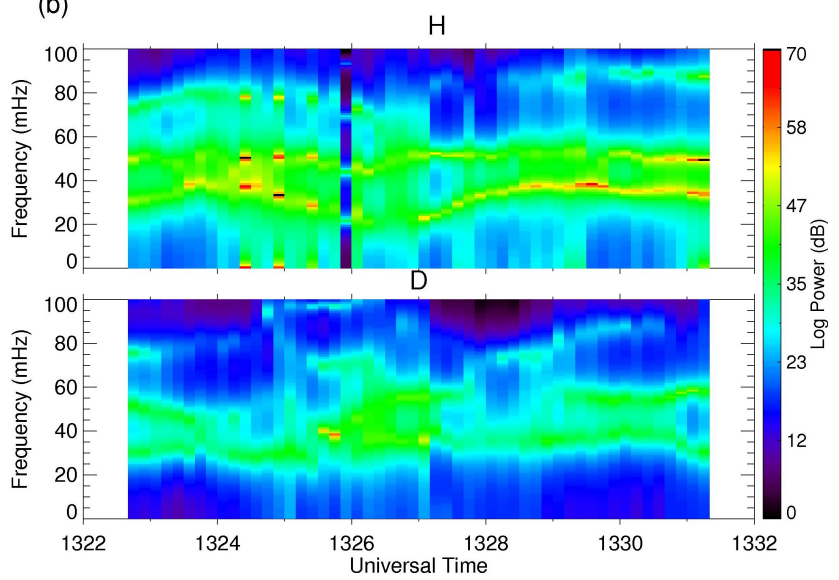

Fig. 4. MESA dynamic power spectra of the HER H- and Dcomponents for (a) data plotted in Fig. 3a and (b) for data plotted in Fig. 3b.

In order to facilitate comparison of the ground and satellite data, dynamic MESA spectra of both data sets were computed using a $90 \mathrm{~s}$ data window shifted by $10 \mathrm{~s}$ as shown in Figs. 4a and 5a for ground and satellite, respectively. The frequency resolution of the dynamic spectra is $1 \mathrm{mHz}$. The prediction error filter (PEF) length values of 15 and 20 determined experimentally (Ndiitwani and Sutcliffe, 2009) were used for ground and satellite data respectively. Additional geocentric latitude and L-value axes are added to the CHAMP dynamic spectra to facilitate studying the L-shell dependence of the field line resonance frequencies. Figure $4 \mathrm{a}$ shows the dynamic spectra computed for the Hermanus ground data. A field line resonance (FLR) is clearly visible in the $\mathrm{H}$-component with frequency centred around $34 \mathrm{mHz}$ between 07:27 UTC and 07:35 UTC. In addition there is an oscillation of much lower power at $65 \mathrm{mHz}$ from 07:29 to 07:32 UTC. The FLR frequency also appears visible in the D-component between 07:30 UTC and 07:35 UTC. In addition, there are weaker bands of oscillation at other frequencies.

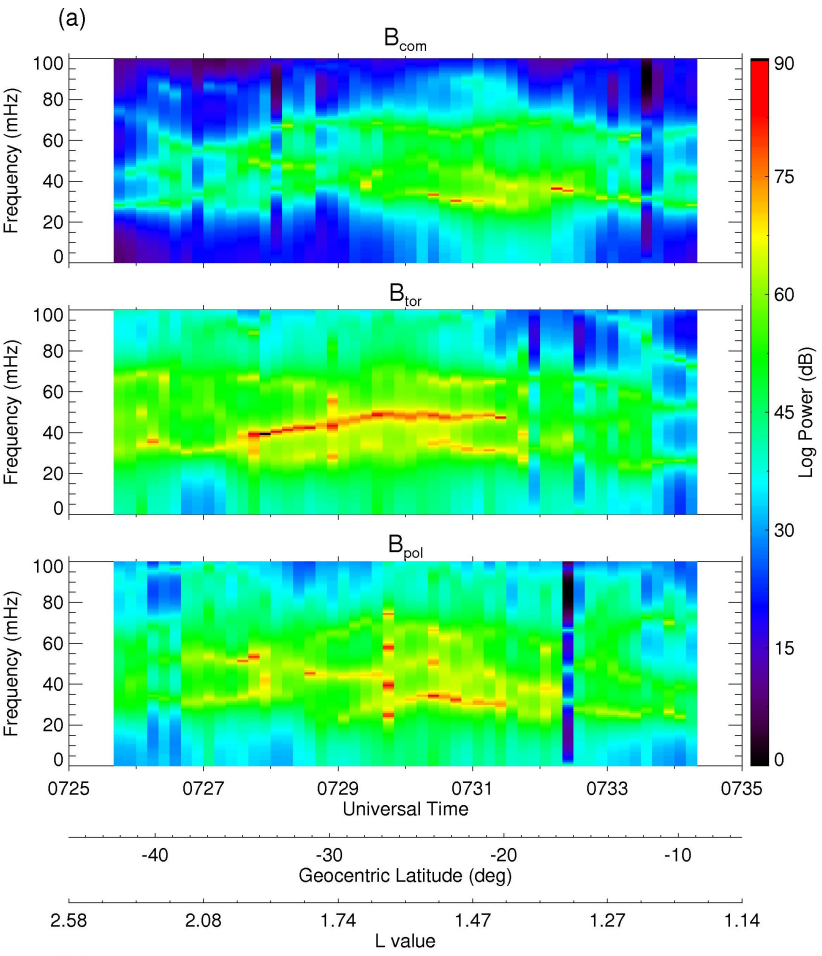

(b)
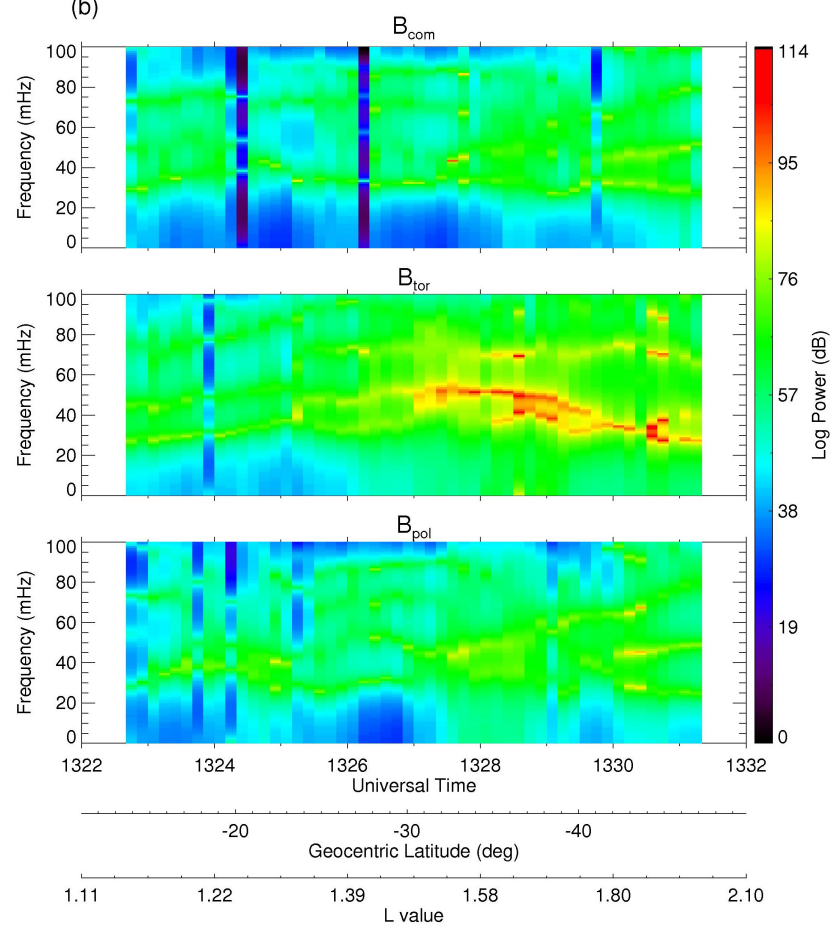

Fig. 5. MESA dynamic power spectra for magnetic components observed along the CHAMP trajectory on (a) 13 February 2002 and (b) 18 February 2003. Also shown are the geocentric latitude and L-shell for the satellite trajectories.

The dynamic spectra of CHAMP data displayed in Fig. 5a exhibit multiple frequency structures that change over the 10min interval of the dynamic spectra. The most noticeable 
aspect observable for all components is increased power in the band of frequencies between about 20 and $70 \mathrm{mHz}$. There are similarities observable between the more intense structures in the compressional $\left(B_{\mathrm{com}}\right)$ and poloidal $\left(B_{\mathrm{pol}}\right)$ components. An important feature in $B_{\text {com }}$ is the broadband frequency structure of enhanced intensity between 30 and $60 \mathrm{mHz}$, increasing to $70 \mathrm{mHz}$, between $07: 26$ and 07:33 UTC.

The most outstanding feature in the toroidal component $\left(B_{\text {tor }}\right)$ is an intense oscillation starting at $30 \mathrm{mHz}$ at about 07:27 UTC that increases to a maximum of $50 \mathrm{mHz}$ at about 07:30 UTC when the satellite reaches a shell value of $L \sim$ 1.6. There are oscillations at $30 \mathrm{mHz}$ and $65 \mathrm{mHz}$ which can be ascribed to coupling with fast mode oscillations. The satellite crossed the latitude of the Hermanus ground station at about 07:28 UTC.

\subsection{Event 2}

The second case study considers the event that occurred between 13:22-13:32 UTC on 18 February 2003. Figure 3b shows 10 min of the HER H- and D-component time series. The signal exhibits a non-wave packet structure with $\mathrm{H}$ component amplitude slightly larger than the D-component. The corresponding satellite signal shown in Fig. 3d exhibits a broadband structure as was the case for the first event considered. The $B_{\text {tor }}$ again exhibits larger amplitude compared to the other two components (note the larger amplitude scale). The prominent feature of the $B_{\text {tor }}$ signal is the increase of amplitude as the satellite moves from low to high latitudes.

The oscillation frequencies of the signals can be viewed from the MESA dynamic spectra shown in Figs. $4 \mathrm{~b}$ and $5 \mathrm{~b}$ for ground and CHAMP signals respectively. In Fig. $4 \mathrm{~b}$ the HER H-component shows two prominent frequency bands of similar intensity. The upper band oscillates at a frequency of about $50 \mathrm{mHz}$. The lower band varies in frequency around $35 \mathrm{mHz}$ going as low 20 and as high as $40 \mathrm{mHz}$. The upper and lower frequency bands are, respectively, associated with FLR and upstream wave (UW) activity. The cross-phase spectrum of the HER-SUT pair (not shown) indicated FLR frequency of 50-55 mHz between 06:00 and 16:00 UTC on this day. This value corresponds to the field line which has its footpoint at the midpoint between HER and SUT. The upstream wave frequency can be estimated from the empirical relationship $f_{\text {uw }}(\mathrm{mHz})=6 \boldsymbol{B}_{\mathrm{IMF}}$ (Yumoto et al., 1984; Russel and Hoppe, 1981). The magnetic field intensity $\boldsymbol{B}_{\mathrm{IMF}}$ observed during the course of this event from the ACE satellite (taking into account the propagation delay from ACE to the magnetopause) was $\sim 6.2 \mathrm{nT}$. Therefore, the value of the empirical relationship $f_{\mathrm{uw}}=\sim 37 \mathrm{mHz}$ is close to the frequency value of the band associated with UW. The solar wind conditions on this day, i.e. high solar wind velocity and small cone angle, were conducive to intense UW activity (B. Heilig, private communication, 2009). The UW activity frequency also appears visible on the D-component lower band that oscil- lates at frequencies between 30 and $40 \mathrm{mHz}$. There is evidence of oscillation at other higher frequencies.

The dynamic spectra for the CHAMP components in Fig. 5b show multiple frequency structures changing over 10 min. This is reminiscent of the first event considered and the Ndiitwani and Sutcliffe (2009) results. The fast mode components, $B_{\mathrm{com}}$ and $B_{\mathrm{pol}}$, exhibit a broadband frequency structure and a number of clear discrete frequency bands around $30,40,75$, and $85 \mathrm{mHz}$. The most outstanding feature of $B_{\text {tor }}$ occurs when the satellite is located between $L=1.3$ and 2.1. There is oscillation centered at $45 \mathrm{mHz}$ around 13:26 UTC when satellite crossed $L \sim 1.4$ and which increases as a function of $\mathrm{L}$-value to a maximum of $52 \mathrm{mHz}$ at 13:28 UTC when the satellite approached $L \sim 1.6$. After the satellite crossed $L \sim 1.6$ the toroidal resonant frequency decreases with increasing L-value to about $27 \mathrm{mHz}$ at 13:31 UTC and $L \sim 2.1$.

\section{Discussion and conclusion}

In the previous section frequency structures, which indicate the presence of L-dependent field line resonance were identified using ground-based and LEO satellite data. In this section the observations are discussed in the light of MHD theoretical models and previously published field line resonance observations. We presented two Pc3 pulsation events characterised by a broadband compressional source spectrum and toroidal resonant oscillations with clear continuous Ldependent frequency.

At low latitudes, plasma density, magnetic field intensity and field line length vary smoothly with latitude. Consequently, the field line resonant frequency is expected to vary smoothly and continuously as a function of latitude. In our results the inner magnetosphere CHAMP observations show this clearly for $L<3$. The resonant field line oscillations are excited where there is a matching driving force, i.e. a fast mode wave. In both events fast mode signatures which exhibit broadband frequency structures able to drive a broad band of FLR toroidal mode oscillations, were observed in the compressional component spectra. In Event 1, the beginning of this behaviour was observed shortly before CHAMP crossed the Hermanus L-shell (i.e. 1.8) with a frequency of $30 \mathrm{mHz}$ observed on CHAMP. In Fig. 5a we see that the FLR frequency in the $B_{\text {tor }}$ spectrum noticeably increased with decreasing latitude, reaching a maximum of $50 \mathrm{mHz}$ when the satellite reached $L \sim 1.6$ and then remained constant. According to the dayside cavity model of Waters et al. (2000), (which invokes the usage of realistic profile of Alfvén velocity of Lee (1996) that depends on the radial coordinate and reaches a maximum at $L=1.6$ ), the FLR frequency increases with decreasing $\mathrm{L}$-value to reach a maximum frequency at a particular L-shell. The frequency then decreases gradually with decreasing $\mathrm{L}$-value due to the increase in plasma density caused by heavy ions such as $\mathrm{O}^{+}$. Menk et al. (2000) 
Table 1. Comparison of the FLR Doppler shifts of previous and current events.

\begin{tabular}{lcccc}
\hline Event & $\begin{array}{c}\text { Ground frequency } \\
(\mathrm{mHz})\end{array}$ & $\begin{array}{c}\text { CHAMP frequency } \\
(\mathrm{mHz})\end{array}$ & Difference & \%Difference \\
\hline Vellante et al. (2004) & 55 & 65 & +10 & 18 \\
Ndiitwani and Sutcliffe (2009) & 45 & 40 & -5 & 11 \\
Event 1 & 34 & 38 & +4 & 12 \\
Event 2 & 50 & 38 & -12 & 24 \\
\hline
\end{tabular}

observed this L-value to be $L \sim 1.6$ in agreement with the cavity model and current results. In our observations the apparent decrease of FLR frequency due to the effect of heavy ions is particularly noticeable in the second case study. In Fig. 5b, before the satellite crossed $L \sim 1.55$ at 13:27.5 UTC, the FLR frequency was observed to increase with the increase in latitude.

When comparing ground and satellite observations, the following was noticed:

In Event 1 the FLR observed on the ground at frequency $34 \mathrm{mHz}$ driven by a compressional wave at the same frequency is observed at $38 \mathrm{mHz}$ in the satellite $B_{\text {tor }}$ spectrum as it crossed the HER L-shell. The difference in frequency is the result of a Doppler frequency shift caused by the rapid movement of the satellite across the resonance region. The frequency shift to higher frequencies is attributed to the equatorward motion of the satellite. In the Ndiitwani and Sutcliffe (2009) example the satellite was moving poleward and the frequency shift was to lower frequencies. Vellante et al. (2004) provides an explanation and derivation of this Doppler shift effect, namely that it is due to the rapid rate at which a LEO satellite traverses the rapid phase change across the field line resonance region. In Event 2, the CHAMP ground track was over the South Atlantic Ocean. Around 13:30 UTC the satellite crossed the $L=1.8 \mathrm{~L}$-shell, where CHAMP observed a frequency of about $38 \mathrm{mHz}$, which is lower than the $50 \mathrm{mHz}$ observed at the HER ground station. The Doppler shift to lower frequency of the satellite FLR frequency with respect ground frequency is the result of the poleward motion of the satellite; however, in this case the frequency difference may also have been affected by the significant longitudinal difference between the satellite track and the Hermanus ground station. In Table 1 the Doppler shifts of previous and current observations are compared. In this table we specify the FLR frequencies observed on the ground and by CHAMP on crossing ground stations L-shells, the difference between the two frequencies, as well their percentage differences. Vellante et al. (2004) observed $18 \%$ difference whereas Ndiitwani and Sutcliffe (2009) observed 11\% difference. In Event 1 a $12 \%$ difference is observed, which is similar to the Ndiitwani and Sutcliffe (2009) observation. A significantly larger percentage difference of $24 \%$ is observed in Event 2. The signs of the Doppler shift are consist and de- pend on the direction of motion of the satellite. The reason for the differences in magnitude is not clear; however, the larger shift for Event 2 may be due to the significant longitudinal difference between the satellite track and the Hermanus ground station.

In summary, we made observations consistent with Hasegawa's (1983) mathematical model of field line resonance excitation and showed examples where $B_{\text {tor }}$ resonates with L-dependent frequency. The examples presented in this study differ from the observations by Vellante et al. (2004) and Ndiitwani and Sutcliffe (2009) who observed discrete FLR frequencies excited at latitudes where there was a matching driving force, i.e. a fast mode wave. The events presented in this paper exhibit toroidal resonant frequency increasing continuously as function of decreasing latitude down to $L \sim 1.6$ and thereafter decreasing as a result of the larger plasma density of the upper ionosphere. This confirms that $\mathrm{Pc} 3$ pulsations do not always have a source with the same frequency structure. The events discussed in this paper arise from a broad band source, while the previous results come from a narrow band source.

Acknowledgements. The satellite data used in this study were obtained from the CHAMP Information System and Data Centre (http: //isdc.gfz-potsdam.de/). We thank A. D. M. Walker and B. Heilig for useful discussions during the course of this work.

Topical Editor R. Nakamura thanks C. L. Waters and another anonymous referee for their help in evaluating this paper.

\section{References}

Baransky, L. N., Borovkov, J. E., Gokhberg, M. B., Krylov, S. M., and Troitskaya, V. A.: High resolution method of direct measurement of the magnetic field line's eigenfrequencies, Planet. Space Sci., 33, 1369-1374, 1985.

Baransky, L. N., Belokris, S. P., Borovkov, Y. E., Gokhberg, M. B., Federov, E. N., and Green, C. A.: Restoration of the meridional structure of geomagnetic pulsation fields from gradient measurements, Planet. Space Sci., 37, 859-864, 1989.

Burg, J. P.: Maximum Entropy Spectral Analysis, Paper presented at 37th Meeting, Soc. Geophys., Oklahoma City, Okla., October, 1967.

Burg, J. P.: A new analysis technique for time series data, Paper presented at NATO Advanced Study Institute on Signal Processing, Enschede, Netherlands, August 1968. 
Chen, L. and Hasegawa, A.: A theory of long-period magnetic pulsations, I: Steady state excitation of field line resonances, J. Geophys. Res., 79, 1024-1032, 1974.

Chen, W. Y. and Stegen, G. R.: Experiment with maximum entropy power spectra of sinusoids, J. Geophys. Res., 79, 3019-3022, 1974.

Dungey, J. W.: Electrodynamics of the outer atmosphere, Ionos. Res. Lab. Sci., University Park, Pa. State Univ., Rep. 69, 1954.

Dungey, J. W.: Evidence of hydromagnetic waves in the Earth's magnetosphere and of their propagation to the Earth's surface, Phys. Rev. Lett., 12, 213-215, 1964.

Engebretson, M. J., Zanetti, L. J., Potemra, T. A., Baumjohann, W., Lühr, H., and Acuna, H.: Simultaneous observation of Pc3-4 pulsations in the solar wind and in the Earth's magnetosphere, J. Geophys. Res., 92, 10053-10062, 1987.

Hasegawa, A., Tsui, K. H., and Assis, A. S.: A theory of long period magnetic pulsations, 3. Local field line oscillations, Geophys. Res. Lett., 10, 765-767, 1983.

Hattingh, S. K. F. and Sutcliffe, P. R.: Pc3 pulsation eigenperiod determination at low latitudes, J. Geophys. Res., 92, 12433-12436, 1987.

Hughes, W. J. and Southwood, D. J.: An illustration of modification of geomagnetic pulsation structure by the ionosphere, J. Geophys. Res., 81, 3241-3247, 1976.

Jacobs, J. A., Kato, Y., Matsushita, S., and Troitskaya, V. A.: Classification of geomagnetic micropulsations, J. Geophys. Res., 69(1), 180-181, 1964.

Le, G. and Russell, C. T.: The morphology of ULF waves in the Earth's Foreshock, in: Solar wind sources of magnetospheric Ultra-Low-Frequency waves, pp. 87-98, Geophysical Monogr. 81, AGU, Washington, D.C., 1994.

Le, G. and Russell, C. T.: Solar wind control of upstream wave frequency, J. Geophys. Res., 101, 2571-2575, 1996.

Lee, D. H.: Dynamics of MHD wave propagation in the low-latitude magnetosphere, J. Geophys. Res., 101, 15371-15386, 1996.

Menk, F. W., Waters, C. L., and Fraser, B. J.: Field line resonances and waveguide modes at low latitudes: 1 . Observations, J. Geophys. Res., 105, 7747-7761, 2000.

Ndiitwani, D. C. and Sutcliffe, P. R.: The structure of low-latitude Pc3 pulsations observed by CHAMP and on the ground, Ann. Geophys., 27, 1267-1277, 2009, http://www.ann-geophys.net/27/1267/2009/.
Russel, C. T. and Hoppe, M. M.: The dependence of upstream wave periods on the interplanetary magnetic field strength, Geophys. Res. Lett., 8, 615-618, 1981.

Shin, K., Kojima, H., Matsumoto, H., and Mukai, T.: Characteristics of electrostatic solitary waves in the Earth's foreshock region: Geotail observations, J. Geophys. Res., 113, A03101, doi:10.1029/2007JA012344, 2008.

Southwood, D. J.: Some features of field line resonances in the magnetosphere, Planet. Space Sci., 22, 483-491, 1974.

Sugiura, M. and Wilson, C. R.: Oscillation of the geomagnetic field lines and associated magnetic perturbations at conjugate points, J. Geophys. Res., 69, 1211-1216, 1964.

Takahashi, K., Anderson, B. J., and Strangeway, R. J.: AMPTE CCE Observations of Pc 3-4 pulsations at $L=2-6$, J.Geophys. Res., 95, 17179-17186, 1990.

Ulrych, T. J. and Clayton, R. W.: Time series modelling and maximum entropy, Phys. Earth Planet. Inter., 12, 188-200, 1967.

Vellante, M., Lühr, H., Zhang, T. L., Wesztergom, V., Villante, U., De Lauretis, M., Piancatelli, A., Rother, M., Schwingenschuh, K., Koren, W., and Magnes, W.: Ground/satellite signatures of field line resonance: A test of theoretical predictions, J. Geophys. Res., 109, A06210, doi:10.1029/2004JA010392, 2004.

Waters, C. L., Menk, F. W., and Fraser, B. J.: The resonance structure of low latitude field line resonances, Geophys. Res. Lett., 18 2293-2296, 1991.

Waters, C. L., Menk, F. W., and Fraser, B. J.: Low-latitude geomagnetic field line resonance: experiment and modeling, J. Geophys. Res., 99, 17547-17558, 1994.

Waters, C. L., Samson, J. C., and Donovan, E. F.: The temporal variation of the frequency of high latitude field line resonances, J. Geophys. Res., 100, 7987-7996, 1995.

Waters, C. L., Harrold, B. G., Menk, F. W., Samson, J. C., and Fraser, B. J.: Field line resonances and waveguide modes at low latitudes 2. A model, J. Geophys. Res., 105, 7763-7774, 2000.

Yumoto, K., Saito, T., Tsurutani, B. T., Smith, E. J., and Akasofu, SI.: Relationship between the IMF magnitude and Pc3 magnetic pulsations in the magnetosphere, J. Geophys. Res., 89, 97319740, 1984. 